\title{
Overexpression of microRNA-196b Accelerates Invasiveness of Cancer Cells in Recurrent Epithelial Ovarian Cancer Through Regulation of Homeobox A9
}

\author{
GUN OH CHONG ${ }^{1}$, HYO-SUNG JEON ${ }^{2}$, HYUNG SOO HAN ${ }^{3}$, JI WOONG SON ${ }^{4}$, YOON HEE LEE ${ }^{1}$, \\ DAE GY HONG ${ }^{1}$, HONG JUN PARK ${ }^{2}$, YOON SOON LEE $^{1}$ and YOUNG LAE CHO ${ }^{1}$ \\ ${ }^{1}$ Department of Obstetrics and Gynecology, School of Medicine, \\ Kyungpook National University Hospital, Daegu, Republic of Korea; \\ ${ }^{2}$ Mmonitor. Inc., Daegu, Republic of Korea; \\ ${ }^{3}$ Department of Physiology, School of Medicine, Kyungpook National University Hospital, Daegu, Republic of Korea; \\ ${ }^{4}$ Department of Internal Medicine, Konyang University Hospital, Daejeon, Republic of Korea
}

\begin{abstract}
Background/Aim: Although microRNAs (miRNAs) are known to influence messenger RNA post-transcriptional control and contribute to human tumorigenesis, little is known about the differences in miRNA expression between primary and recurrent epithelial ovarian cancer (EOC). The purpose of this study was to assess the differential miRNA expression between primary and recurrent EOC and to investigate whether miR-196b could regulate the expression of the Homeobox A9 (HOXA9) gene, and thus affect the invasiveness of cancer cells in recurrent EOC. Materials and Methods: Microarrays were used to generate the expression profiles of 6658 miRNAs from samples of 10 patients with EOC. miRNA expression patterns were compared between primary and recurrent EOC. Aberrantly expressed miRNA, associated genes, and invasion activities were validated by a luciferase assay and an in vitro invasion assay. Results: miRNA microarray analysis identified 33 overexpressed miRNAs (including miR-196b) and 18 under expressed miRNAs in recurrent EOC from 6658 human miRNAs. HOXA9 expression was inversely correlated with miR-196b levels in recurrent EOC. We noted that miR-196b induced
\end{abstract}

This article is freely accessible online.

Correspondence to: Prof. Young Lae Cho, M.D. Kyungpook National University Medical Center, Daegu, Korea, 807 Hogukno, Buk-gu, 702-210 Daegu, Republic of Korea. Tel: +82 532002681, Fax: +82 532002028, e-mail: ylchoknuh@naver.com and Dr. HyoSung Jeon, Ph.D. Mmonitor Inc., Daegu, Korea, Seongseogongdanro 11-gil, Dalseo-gu, Daegu, Republic of Korea. Tel: +82 532542505, Fax: +82 532542507, e-mail: jeonh@mmonitorings.com

Key Words: Epithelial ovarian cancer, recurrence, invasiveness, miR-196b, HOXA9 gene. ovarian cancer cell invasiveness in recurrent EOC by an in vitro invasion assay. Conclusion: Overexpression of $m i R$ $196 b$ may contribute to invasion activities in recurrent EOC by regulating the HOXA9 gene. Moreover, miR-196b can be a potential biomarker in recurrent EOC.

Epithelial ovarian cancer (EOC) is the most lethal of all gynecological cancers, the fourth leading cause of cancerrelated deaths in women in the United States, and the fifth most common malignancy in women in developed countries (1). In general, less than half $(45 \%)$ of EOC patients can survive for more than five years after the initial diagnosis (2). The poor survival from EOC is due to the high percentage of patients diagnosed at an advanced stage, who often develop resistance to combined chemotherapy and show substantially poor prognosis.

Most patients with EOC are treated with platinum- and taxane-based chemotherapy. Although initial treatment is successful for $80-90 \%$ patients, most responders eventually acquire resistance to a wide range of chemotherapeutic agents. Prediction of patients that respond to a distinct therapy would help to optimize tailored treatment.

MicroRNAs (miRNAs) are small ( 22 nucleotides) noncoding RNAs that regulate gene expression at the transcriptional and/or post-transcriptional levels (3). These molecules typically reduce the translation and stability of messenger RNA (mRNA), including that of genes mediating processes in tumorigenesis such as inflammation, cell cycle regulation, stress response, differentiation, apoptosis, and invasion. miRNA targeting is initiated through specific base-pairing interactions between the 5 ' end ("seed" region) of the miRNA and sites within the coding and untranslated regions (UTRs) of mRNAs; target sites in the 3'-UTR lead to more effective mRNA destabilization (4). miRNAs play a role in the tumorigenic process and are potential 
therapeutic targets and novel biomarkers in most human cancers. Moreover, numerous miRNA profiling studies in EOC have identified miRNAs associated with chemotherapy resistance and disease progression (5-8). However, little is known about the differences in miRNA expression between primary and recurrent EOC. Previously, we reported aberrant miRNA expression in recurrent EOC compared to that in primary EOC. To understand the biology of recurrent ovarian cancer, we examined the expression of 6658 miRNAs in both primary and recurrent EOC samples. Among 6,658 human miRNAs, 33 miRNAs were overexpressed and 18 miRNAs were under expressed in recurrent EOC (9).

The purpose of this study was to investigate the expression level of miRNAs in recurrent EOC and to examine the expression levels of key components of the miRNA processing machinery.

\section{Materials and Methods}

Patients and tissue samples. Between September 2013 and May 2014, tumor tissue specimens were obtained from 10 Korean patients with EOC (primary EOC, $n=5$; recurrent EOC, $n=5$ ) who underwent surgery at the Kyungpook National University Medical Center, Daegu, Korea. Histopathologic diagnoses were established using the World Health Organization criteria, and the tumor histotype was determined as serous cystadenocarcinoma in all patients. The primary and recurrent EOC cases were different patient populations. The 5 patients with recurrent EOC had received at least 6 cycles of platinum-based combination chemotherapy (paclitaxel plus carboplatin). Tissue specimens were obtained during surgery, and they were rapidly frozen in liquid nitrogen and stored at $-80^{\circ} \mathrm{C}$ until analysis. Tissue samples were histologically confirmed by hematoxylin-eosin staining. The Institutional Review Board approved the study protocol, and written informed consent was obtained from all patients.

miRNA microarray. Total RNA $(2 \mu \mathrm{g})$ was extracted from the transduced cells using Trizol and the RNeasy Miniprep kit (Qiagen, Hilden, Germany) according to the manufacturers' protocols. The RNA quality was verified with the Agilent RNA Bioanalyzer (Agilent Technologies, Palo Alto, CA, USA). Biotinylated cRNA was amplified using double in vitro transcription in accordance with the Affymetrix small sample labeling protocol VII (Affymetrix, Santa Clara, CA, USA). Total RNA was then hybridized onto an Affymetrix GeneChip ${ }^{\circledR}$ miRNA 4.0 as per standard protocols of the provider. Fluorescence intensities were quantified and analyzed using the Genechip operating software (Affymetrix). Raw data were normalized by the Robust Multi-array Average (RMA) method to remove systematic variations. Briefly, RMA corrects raw data for background by using a formula based on normal distribution and uses a linear model to estimate values on a log-scale. Transcripts whose logtransformed expression ratios differed by at least two-folds between recurrent and primary EOC tissues, were identified.

Cell culture and growth conditions. Ovarian cancer cell lines SK-OV3 and 293T were purchased from American Typical Culture Collection $\left(\mathrm{ATCC}^{\circledR}, \mathrm{HTB}-77^{\mathrm{TM}}, \mathrm{CRL}-3216^{\mathrm{TM}}\right.$, respectively, Manassas, VA, USA) and were maintained in DMEM (high glucose)

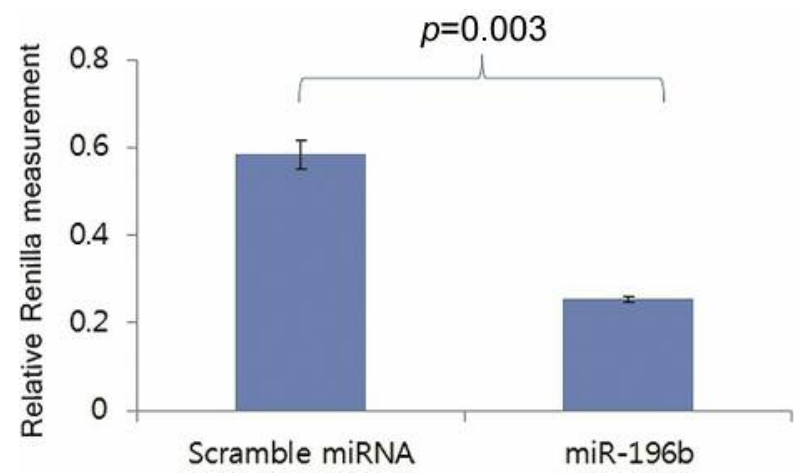

Figure 1. Relative Renilla luciferase activity.

(Gibco ${ }^{\circledR}$, Rockville, MD, USA) with $10 \%$ fetal bovine serum (FBS) and antibiotics (penicillin 100 units/ml and streptomycin $100 \mathrm{mg} / \mathrm{ml}$ ).

Construction of plasmid and luciferase assay. To investigate whether miR-196b modulates HOXA9 expression by binding its 3'UTR, a luciferase assay was performed using psiCHECK2 plasmid containing the HOXA9 3'-UTR. The 1,035 bp fragment of HOXA9 3'-UTR was synthesized by PCR and cloned into a dual luciferase vector, psiCHECK2 (Promega, Madison, WI, USA). The forward primer with a XhoI restriction site (5'-CCA CTC GAG AAA GAA CTG TCC GTC CCC CT-3') and the reverse primer with the NotI restriction site (5'-CCA GCG GCC GCG GTC AGT AGG CCT TGA GGT AAC-3') were used to amplify the HOXA9 3'-UTR region. The correct sequence of the clone was verified by DNA sequencing. The $293 \mathrm{~T}$ cells were seeded in 12-well plates in DMEM supplemented with $10 \%$ heat-inactivated FBS and then transfected with psiCHECK2-HOXA9 constructs containing the 3'-UTR of HOXA9, in the presence of a miR-196b mimic or scrambled miRNA (Ambion) using the Effectene (Qiagen, Hilden, Germany) transfection reagent. The cells were harvested at 48 hours after transfection and the cell lysates were prepared according to Promega instruction manual. Renilla luciferase activity was measured using a Lumat LB953 luminometer (EG \& G Berthhold, Bad Wildbad, Germany), and the results were normalized using luciferase activity. All experiments were performed in triplicate.

In vitro invasion assays. The invasion assay was performed in triplicate using 48-well microchemotaxis chambers (Neuro. Probe, Inc., Gaithersburg, MD, USA) with 8 - $\mu$ m pore membranes (Neuro. Probe, Inc., Gaithersburg, MD, USA) pre-coated with $10 \mu \mathrm{g} / \mathrm{ml}$ Matrigel (BD Bioscience). SK-OV-3 cells $\left(1 \times 10^{4}\right)$ in $50 \mu \mathrm{l}$ of serum-free medium were seeded in the upper chamber, and the lower chamber was filled with $26-27 \mu \mathrm{l}$ medium containing $10 \%$ FBS. After incubation for $24 \mathrm{~h}$ at $37^{\circ} \mathrm{C}$, cells that migrated to the lower surface of the membranes were stained with a Diff-Quick kit (Sysmex, Kobe, Japan) and then counted under a microscope. The chambers were stained with $0.2 \%$ crystal violet and analyzed by photography. The migration assay was performed with the same procedure using membranes coated with $5 \mu \mathrm{g} / \mathrm{ml}$ collagen IV (TRAVIGEN, Gaithersbug, MD, USA).

Statistical analysis. For Genechip microarray analysis, statistical comparisons were made using the Student's $t$-test. Differentially 
A

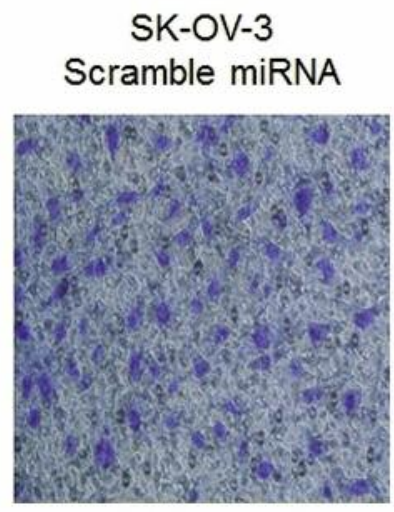

SK-OV-3

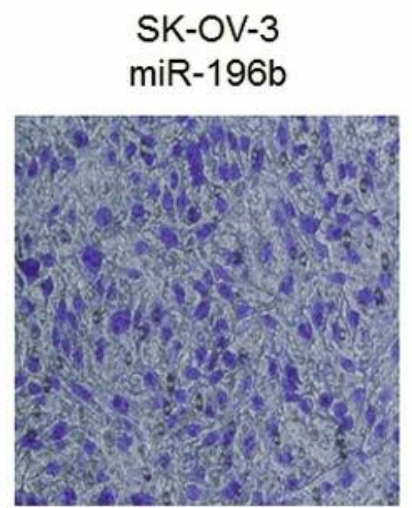

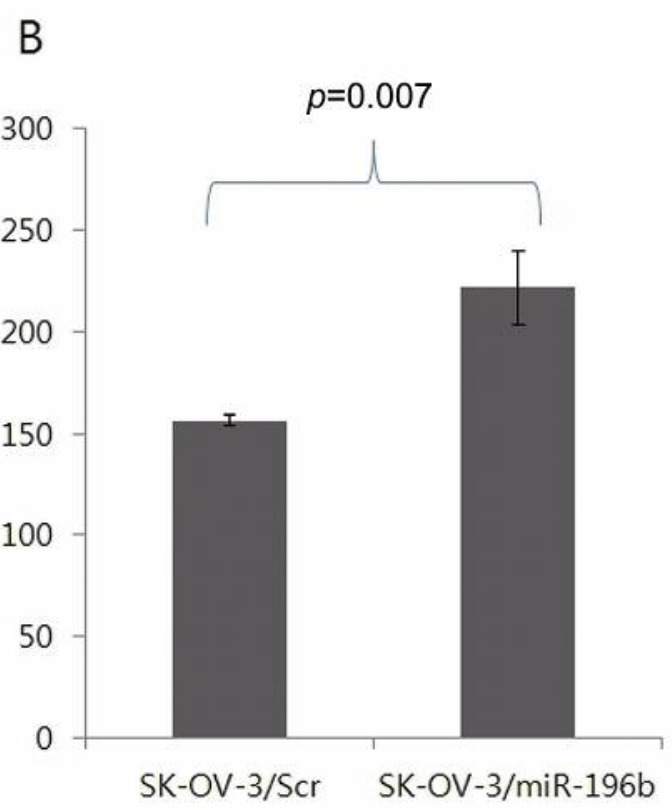

Figure 2. Increased SK-OV-3 cell invasion by miR-196b transfection. (A) Crystal violet (0.2\%) staining of invading cells in the upper chamber. (B) Number of invading cells.

expressed miRNAs between primary and recurrent EOC were detected through the $t$-test and data were considered statistically significant when $p<0.05$.

\section{Results}

Selection of biomarkers of recurrent EOC. In the Affymetrix miRNA 4.0 analysis, 4 miRNAs including miR551b, miR-19b, miR-196b, and miR-3198, were significantly overexpressed in recurrent EOC from among 6,658 human miRNAs. We chose miR-196b for further studies because it was suggested to be a potential marker in patients with lung adenocarcinoma (10) and in gastric and oral cancer $(11,12)$. However, the role of miR-196b in EOC is not fully investigated. The result of the microarray analysis showed that miR-196b transcription level was significantly higher in recurrent EOC than that in primary EOC. The expression level of miR-196b was 138.5 (average score) in recurrent EOC and 6.5 (average score) in primary $\operatorname{EOC~}(p=0.031)$.

Repression of HOXA9 transcription by miR-196b. To identify a direct target gene of miR-196b, we searched public databases such as TagetScan and then selected HOXA9 from the top 10 target genes including HOXA7, HOXC8, SMC3, SLC9A6, HOXA9, HMGA2, CTBS, ZMYND11, Clarf88, and COL16A1. In the female reproductive system, HOXA9, HOXA10, HOX11, and HOXA13 are expressed along the Müllerian duct axis (13). Especially, HOXA9 is expressed in the fallopian tubes and has a functional role in normal development and in adult tissues, as well as in cancer (14). Therefore, HOXA9 might be a candidate target of miR-196b. To verify whether miR-196b could regulate the HOXA9 gene directly, we generated a Renilla luciferase reporter plasmid cloned downstream of the HOXA9 3'-UTR containing the putative miR-196b binding sequences, which was predicted to contain two binding sites, position 940-959 bp and 1973-1995 of HOXA9 3'-UTR. The constructs were then co-transfected into 293T cells along with miR-196b, or scrambled miRNA, and Renilla luciferase activity was measured after $48 \mathrm{~h}$. As shown in Figure 1, Renilla activity with the miR-196b mimic was significantly lower than that with scrambled miRNA $(p=0.003)$. This suggests that miR196b represses HOXA9 transcription by directly binding to its 3'-UTR.

Induction of cancer cell invasion by miR-196b. To determine whether miR-196b has an effect on ovarian cancer progression, an invasion assay was performed with SK-OV-3 ovarian cancer cells in vitro. miR-196b was significantly overexpressed in recurrent EOC. The miR196b mimic and scrambled miRNA were transfected into SK-OV3 ovarian cancer cells. The invasion assays indicated that overexpression of miR-196b could significantly induce invasiveness in SK-OV-3 cells (Figure 2), suggesting that HOXA9 might be involved in inhibiting aggressive behavior in recurrent EOC. 


\section{Discussion}

Management of EOC has improved over the last 20 years due to effective surgery and optimized combinational chemotherapy, i.e. platinum-based drugs combined with taxanes (15). However, the overall cure rate is only $30 \%$. The issues of tumor recurrence, drug resistance, enhanced invasion, and metastasis remain a challenge in the treatment and clinical management of EOC. While existing therapies are considered relatively effective in the treatment of ovarian tumors, mesenchymal/stem cell-like metastasizing EOC cells are generally resistant to these therapies and are considered largely responsible for EOC recurrence (16-18). Several miRNAs are involved in response to chemotherapy. Low levels of miR-200c have been associated with a mesenchymal phenotype and may thus be associated with chemoresistance (19). Furthermore, low levels of miR-199a may be a reliable predictor for chemoresistance in recurrent tumors (7). These miRNAs can affect the response to chemotherapeutic drugs and might have both prognostic, as well as predictive value. However, the mechanism of tumor recurrence and chemoresistance in EOC remains poorly understood. Moreover, only a few studies have reported the differential miRNA profiles between primary and recurrent EOC. Laios et al. demonstrated that miR-9 and miR-223 could be of potential importance as biomarkers in recurrent EOC (20).

To understand the biology of recurrent EOC and evaluate recurrent EOC-specific biomarkers, we investigated the expression of miRNAs in primary and recurrent EOC. Among the overexpressed miRNAs, miR-19b has a role in oncogenic progression via targeting TP53 $(11,21,22)$. miR$551 \mathrm{~b}$ and miR-3198 were poorly discovered with tumorigenesis. miR-196b was demonstrated to have oncogenic functions in many cancers except ovarian cancer. miR-196b promotes invasiveness in oral cancer through the NME4-JNK-TIMP1-MMP signaling pathway (11), and miR196 b overexpression in gastric cancer was correlated with poor prognosis (23).

The homeotic or HOX genes share a common 120 base pair DNA sequence or the homeobox, which encodes a 61-amino acid peptide called the homeodomain. HOX genes are involved in the development of various cancers. HOX genes act not only as transcriptional activators in cancers but also as transcriptional repressors. Thus, both up-regulation and downregulation of the members of the HOX family of transcription factors appear critical for the promotion of tumorigenesis (24). Aberrant expression of HOX genes has been reported in various cancers including EOC. Ko et al. reported that HOXA9 stimulated the ability of EOC cells to attach to peritoneal cells and to migrate. Thus, HOXA9 contributes to poor outcomes by promoting intraepithelial dissemination via induction of P-cadherin (25). However, HOXA9 was expressed at lower levels in cancerous tissues compared to normal tissues in the context of breast cancer (26). Recent review articles demonstrated that HOX genes are essential regulators of tissue identity and drive tumorigenesis and progression through their involvement in regulating processes such as differentiation, proliferation, adhesion, migration, and apoptosis in EOC (27). Our data revealed that HOXA9 downregulation led to invasiveness by miR-196b in EOC. Thus, it is not yet known whether HOXA9 functions as an oncogene or as a tumor suppressor, and its role in ovarian tumorigenesis and progression is not yet identified. Genomic analysis of tumors from large cohorts of EOC patients with documented treatment and outcome data are needed to further elucidate the mechanisms underlying HOX gene dysregulation, establish their functional prognostic and predictive roles in EOC development, and replicate previous clinical findings.

In the present study, 21 miRNAs were overexpressed and 16 miRNAs were under expressed in recurrent EOC. HOXA9 was directly regulated by miR-196b through binding of its 3'-UTR. miR-196b was overexpressed in recurrent EOC and induced ovarian cancer cell invasiveness. Based on these data, miR-196b can be used a biomarker predicting EOC recurrence. HOXA9 plays a central role in controlling the aggressive behavior of recurrent EOC.

\section{Conflicts of Interest}

The Authors declare that there are no conflicts of interest.

\section{Acknowledgements}

This work was supported by the Technological Innovation R\&D Program (S2260851) funded by the Small and Medium Business Administration (SMBA, Korea).

\section{References}

1 Jemal A, Bray F, Center MM, Ferlay J, Ward E and Forman D: Global cancer statistics. CA Cancer J Clin 61: 69-90, 2011.

2 Jemal A, Siegel R, Ward E, Hao Y, Xu J, Murray T and Thun MJ: Cancer statistics, 2008. CA Cancer J Clin 58: 71-96, 2008.

3 Bartel DP: MicroRNAs: genomics, biogenesis, mechanism, and function. Cell 116: 281-297, 2004.

4 Farazi TA, Hoell JI, Morozov P and Tuschl T: MicroRNAs in human cancer. Adv Exp Med Biol 774: 1-20, 2013.

5 Mateescu B, Batista L, Cardon M, Gruosso T, de Feraudy Y, Mariani O, Nicolas A, Meyniel JP, Cottu P, Sastre-Garau X and Mechta-Grigoriou F: miR-141 and miR-200a act on ovarian tumorigenesis by controlling oxidative stress response. Nat Med 17: 1627-1635, 2011.

6 Iorio MV, Visone R, Di Leva G, Donati V, Petrocca F, Casalini P, Taccioli C, Volinia S, Liu CG, Alder H, Calin GA, Ménard S and Croce CM: MicroRNA signatures in human ovarian cancer. Cancer Res 67: 8699-8707, 2007.

7 Nam EJ, Yoon H, Kim SW, Kim H, Kim YT, Kim JH, Kim JW and Kim S: MicroRNA expression profiles in serous ovarian carcinoma. Clin Cancer Res 14: 2690-2695, 2008. 
8 Yang N, Kaur S, Volinia S, Greshock J, Lassus H, Hasegawa K, Liang S, Leminen A, Deng S, Smith L, Johnstone CN, Chen XM, Liu CG, Huang Q, Katsaros D, Calin GA, Weber BL, Bützow R, Croce CM, Coukos G and Zhang L: MicroRNA microarray identifies Let-7i as a novel biomarker and therapeutic target in human epithelial ovarian cancer. Cancer Res 68: 1030710314, 2008.

9 Chong GO, Jeon HS, Han HS, Son JW, Lee YH, Hong DG, Lee YS and Cho YL: Differential MicroRNA expression profiles in primary and recurrent epithelial ovarian cancer. Anticancer Res 35: 2611-2617, 2015.

10 Yu SL, Lee DC, Sohn HA, Lee SY, Jeon HS, Lee JH, Park CG, Lee HY, Yeom YI, Son JW, Yoon YS and Kang J: Homeobox A9 directly targeted by miR-196b regulates aggressiveness through nuclear Factor-kappa B activity in non-small cell lung cancer cells. Mol Carcinog 55: 1915-1926, 2016.

$11 \mathrm{Lu}$ YC, Chang JT, Liao CT, Kang CJ, Huang SF, Chen IH, Huang CC, Huang YC, Chen WH, Tsai CY, Wang HM, Yen TC You GR, Chiang CH and Cheng AJ: OncomiR-196 promotes an invasive phenotype in oral cancer through the NME4-JNKTIMP1-MMP signaling pathway. Mol Cancer 13: 218, 2014.

12 Liao YL, Hu LY, Tsai KW, Wu CW, Chan WC, Li SC, Lai CH, Ho MR, Fang WL, Huang KH and Lin WC: Transcriptional regulation of miR-196b by ETS2 in gastric cancer cells. Carcinogenesis 33: 760-769, 2012.

13 Taylor HS, Vanden Heuvel GB and Igarashi P: A conserved Hox axis in the mouse and human female reproductive system: late establishment and persistent adult expression of the Hoxa cluster genes. Biol Reprod 57: 1338-1345,1997.

14 Cheng W, Liu J, Yoshida H, Rosen D and Naora H: Lineage infidelity of epithelial ovarian cancers is controlled by HOX genes that specify regional identity in the reproductive tract. Nat Med 11: 531-537, 2005.

15 Cannistra SA: Cancer of the ovary. N Engl J Med 351: 2519-29, 2004.

16 Zhang S, Balch C, Chan MW, Lai HC, Matei D, Schilder JM, Yan PS, Huang TH and Nephew KP: Identification and characterization of ovarian cancer-initiating cells from primary human tumors. Cancer Res 68: 431143-20, 2008.

17 Wang L, Mezencev R, Bowen NJ, Matyunina LV and McDonald JF: Isolation and characterization of stem-like cells from a human ovarian cancer cell line. Mol Cell Biochem 363: 257-268, 2012.

18 Alvero AB, Chen R, Fu HH, Montagna M, Schwartz PE, Rutherford T, Silasi DA, Steffensen KD, Waldstrom M, Visintin I and Mor G: Molecular phenotyping of human ovarian cancer stem cells unravels the mechanisms for repair and chemoresistance. Cell Cycle 8: 158-166, 2009
19 Cochrane DR, Spoelstra NS, Howe EN, Nordeen SK and Richer JK: MicroRNA-200c mitigates invasiveness and restores sensitivity to microtubule-targeting chemotherapeutic agents. Mol Cancer Ther 8: 1055-1066, 2009.

20 Laios A, O'Toole S, Flavin R, Martin C, Kelly L, Ring M, Finn SP, Barrett C, Loda M, Gleeson N, D'Arcy T, McGuinness E, Sheils O, Sheppard B and O' Leary J: Potential role of miR-9 and miR-223 in recurrent ovarian cancer. Mol Cancer 7: 35, 2008.

21 Fan Y, Yin S, Hao Y, Yang J, Zhang H, Sun C, Ma M, Chang Q and Xi JJ: miR-19b promotes tumor growth and metastasis via targeting TP53. RNA 20: 765-772, 2014.

22 Lepore I, Dell'Aversana C, Pilyugin M, Conte M, Nebbioso A, De Bellis F, Tambaro FP, Izzo T, Garcia-Manero G, Ferrara F, Irminger-Finger I and Altucci L: HDAC inhibitors repress BARD1 isoform expression in acute myeloid leukemia cells via activation of miR-19a and/or b. PLoS One 8: e83018, 2013.

23 Lim JY, Yoon SO, Seol SY, Hong SW, Kim JW, Choi SH, Lee JS and Cho JY: Overexpression of miR-196b and HOXA10 characterize a poor-prognosis gastric cancer subtype. World J Gastroenterol 19: 7078-7088, 2013.

24 Bhatlekar S, Fields JZ and Boman BM: HOX genes and their role in the development of human cancers. J Mol Med (Berl) 92: 811-823, 2014.

25 Ko SY and Naora H: HOXA9 promotes homotypic and heterotypic cell interactions that facilitate ovarian cancer dissemination via its induction of P-cadherin. Mol Cancer 13: $170,2014$.

26 Makiyama K, Hamada J, Takada M, Murakawa K, Takahashi Y, Tada M, Tamoto E, Shindo G, Matsunaga A, Teramoto K, Komuro K, Kondo S, Katoh H, Koike T and Moriuchi T: Aberrant expression of HOX genes in human invasive breast carcinoma. Oncol Rep 13: 673-679, 2005.

27 Miller KR, Patel JN, Ganapathi MK, Tait DL and Ganapathi RN: Biological role and clinical implications of homeobox genes in serous epithelial ovarian cancer. Gynecol Oncol 141: 608-615, 2016.
Received January 11, 2017

Revised February 23, 2017

Accepted February 24, 2017 